\title{
Pharmacokinetic and pharmacogenetic determinants of the activity and toxicity of irinotecan in metastatic colorectal cancer patients
}

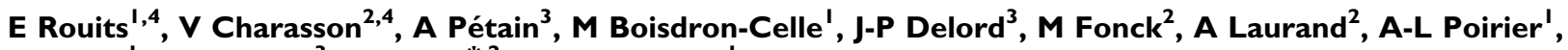 \\ A Morel', E Chatelut ${ }^{3}$, J Robert ${ }^{*, 2}$ and E Gamelin' \\ 'Laboratoire d'Oncopharmacologie, Centre Paul-Papin, 2 rue Moll, Angers 49000, France; ²Département de Pharmacologie INSERM U9I6, Institut \\ Bergonié, 229 cours de l'Argonne, Bordeaux 33076, France; '́quipe d'accueil 3035, Université de Toulouse, Institut Claudius-Regaud, \\ 20 rue du Pont Saint-Pierre, Toulouse 31052, France
}

\begin{abstract}
This study aims at establishing relationships between genetic and non-genetic factors of variation of the pharmacokinetics of irinotecan and its metabolites; and also at establishing relationships between the pharmacokinetic or metabolic parameters and the efficacy and toxicity of irinotecan. We included 49 patients treated for metastatic colorectal cancer with a combination of 5-fluorouracil and irinotecan; a polymorphism in the UGTIAI gene (TA repeat in the TATA box) and one in the CES2 gene promoter $(830 \mathrm{C}>\mathrm{G})$ were studied as potential markers for SN-38 glucuronidation and irinotecan activation, respectively; and the potential activity of CYP3A4 was estimated from cortisol biotransformation into $6 \beta$-hydroxycortisol. No pharmacokinetic parameter was directly predictive of clinical outcome or toxicity. The AUCs of three important metabolites of irinotecan, SN-38, SN-38 glucuronide and APC, were tentatively correlated with patients' pretreatment biological parameters related to drug metabolism (plasma creatinine, bilirubin and liver enzymes, and blood leukocytes). SN-38 AUC was significantly correlated with blood leukocytes number and SN-38G AUC was significantly correlated with plasma creatinine, whereas APC AUC was significantly correlated with plasma liver enzymes. The relative extent of irinotecan activation was inversely correlated with SN-38 glucuronidation. The TATA box polymorphism of UGTIAI was significantly associated with plasma bilirubin levels and behaved as a significant predictor for neutropoenia. The level of cortisol $6 \beta$-hydroxylation predicted for the occurrence of diarrhoea. All these observations may improve the routine use of irinotecan in colorectal cancer patients. UGTIAI genotyping plus cortisol $6 \beta$-hydroxylation determination could help to determine the optimal dose of irinotecan.

British Journal of Cancer (2008) 99, 1239- 1245. doi: I0.1038/sj.bjc.6604673 www.bjcancer.com
\end{abstract}

Published online 16 September 2008

(c) 2008 Cancer Research UK

Keywords: colorectal cancer; irinotecan; pharmacokinetics; UGTIAI gene polymorphism; CYP3A4

Colorectal adenocarcinoma is a leading cause of cancer in developed countries and is responsible for 16000 deaths every year in France (Hill and Doyon, 2005) and 200000 in Europe (Boyle and Ferlay, 2005). This is a curable disease as long as metastatic dissemination has not occurred, and even in that case, the combination of chemotherapy and surgical removing of hepatic metastases is sometimes able to cure the patient (Bentrem et al, 2005). Colorectal cancer was considered 20 years ago as a globally chemoresistant disease and the only available drug was 5-fluorouracil (5-FU), which, however, provided low response rates and limited survival enhancement. Progress came first from a better understanding of 5-FU pharmacology: the combination with folinic acid and the prolongation of drug infusion have considerably improved the efficiency of single-drug 5-FU therapy (Labianca et al, 1997). In addition, the discovery of several major active drugs, namely irinotecan and oxaliplatin, has completely modified our perception of chemosensitivity of colorectal cancer (Douillard et al, 2000; de Gramont et al, 2000). Palliative treatment

\footnotetext{
*Correspondence: Dr J Robert; E-mail: robert@bergonie.org

${ }^{4}$ These authors contributed equally to this work.

Received 10 July 2008; revised 14 August 2008; accepted 22 August 2008; published online 16 September 2008
}

of metastatic colorectal cancer is now based on the combination of folinic acid-modulated 5-FU (or another thymidylate synthase inhibitor) with either irinotecan or oxaliplatin, and allows to obtain response rates in the range of $40-60 \%$ with an overall survival benefit of more than 24 months (Taieb et al, 2005). This success has encouraged the use of such combinations in the adjuvant setting. Finally, the introduction of therapeutic antibodies directed against the EGF receptor or VEGF has further refined the treatment protocols (Cunningham et al, 2004; Moses et al, 2004).

The choice of the chemotherapy regimen appears critical: in view of the impressive survival advantage that can be expected in the palliative setting, and in view of the possible curability, it is crucial to offer to every patient the maximum likelihood of drug efficacy. We have currently no rational way to choose one combination over the other(s), in particular no predictive test allowing to select irinotecan or oxaliplatin to be combined with 5-FU. If there is some drug selectivity, it would be of utmost interest to identify the parameters that determine this selectivity to prescribe the drug or drug combination the most likely to provide a response and a survival advantage to a given patient. In addition, it would be also important to predict for drug-associated toxicity to avoid the prescription of a drug with a high risk of neutropoenia, diarrhoea or mucosal damage. Furthermore, we need to identify individual parameters that are predictive of drug-induced toxicity. 


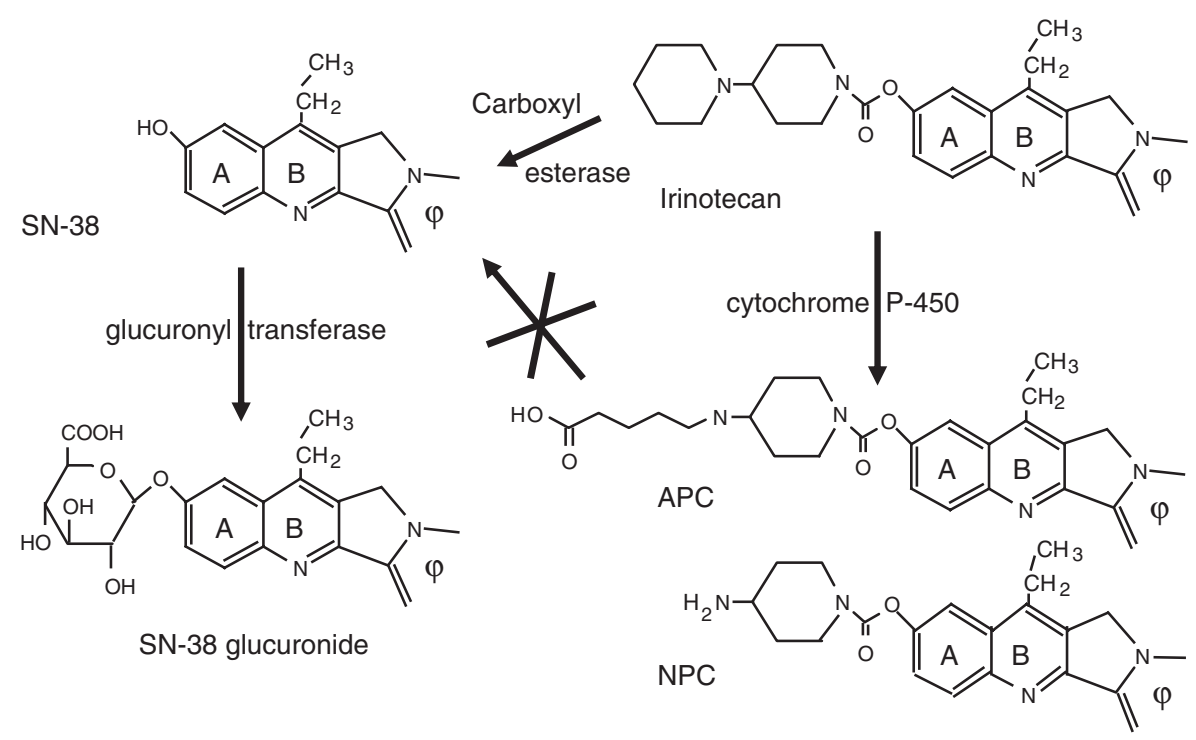

Figure I The metabolism of irinotecan in humans.

The pharmacology of irinotecan (Figure 1) is rather complex (Rivory and Robert, 1995) for several reasons: (i) it is a prodrug that needs activation to a metabolite, $\mathrm{SN}-38$, to interact with its target, DNA-topoisomerase I (Top1); this activation, which is catalysed by carboxylesterase 2 (CES2), is believed to mainly occur in the liver, but in situ activation in the tumour cell cannot be ruled out; (ii) irinotecan and $\mathrm{SN}-38$ are subject to metabolism to inactive species: irinotecan is detoxified by cytochrome $\mathrm{P} 450$ CYP3A4 to APC and NPC, whereas $\mathrm{SN}-38$ is conjugated to glucuronic acid by UDP-glucuronosyl transferase UGT1A1 and possibly other isoforms; (iii) all camptothecins derivatives and metabolites are subject to spontaneous interconversion between a lactone (active) form and a carboxylate (inactive) form, depending on the $\mathrm{pH}$ of the fluid. Several enzymes involved in irinotecan metabolism present individual variations that may be of genetic origin: it is well known that the CYP3A4 liver concentrations may vary in a $1: 20$ ratio, which has been shown to depend on environmental factors rather than on gene polymorphisms (Lamba et al, 2002); furthermore, UGT1A1 promoter is subject to a frequent polymorphism, leading to decreased activity, low SN-38 detoxification and increased risk for irinotecan toxicity (Iyer et al, 2002); finally, little is known about the polymorphisms of CES2 but a polymorphism occurring in the promoter might well be responsible for decreased enzyme expression (Charasson et al, 2004).

We wanted in this study to establish the relationships that may exist between the genetic (gene polymorphisms) and non-genetic (CYP3A4 status) factors of variation of the metabolic transformations of irinotecan and the pharmacokinetics of the drug and its metabolites; and, further, to establish relationships between the pharmacokinetic or metabolic parameters and the efficacy and toxicity of irinotecan. A number of biological parameters potentially indicative of the drug elimination ability of the patients (plasma creatinine, bilirubin and liver enzymes) were studied at the onset of the treatment for studying their association with the pharmacokinetic parameters. This was achieved in a group of 49 patients treated for metastatic colorectal cancer with a combination of 5-FU and irinotecan; the pharmacokinetics of irinotecan and its metabolites were studied in all patients; the polymorphisms of UGT1A1 and CES2 were studied as potential markers for SN-38 glucuronidation and irinotecan activation, respectively; and the potential activity of CYP3A4 was estimated from cortisol biotransformation into $6 \beta$-hydroxycortisol (Yamamoto et al, 2000).

\section{PATIENTS AND METHODS}

\section{Patients}

The patients included in this study suffered from advanced or metastatic colorectal cancer, which had been histologically proven, and had never been treated with irinotecan. They could have received folinic acid-modulated 5-FU treatment as adjuvant or palliative therapy. Patients had to be between 18 and 85 years, with normal biochemical and haematological tests and a performance status $\leqslant 2$, evaluated as defined by the World Health Organization (WHO). They were followed regularly all along the evolution of the disease, and computed tomography scans were performed every 2 months for the evaluation of drug response and event-free survival. They were treated with the FOLFIRI regimen (André et al, 1999), and their treatment included folinic acid $400 \mathrm{mg} \mathrm{m}^{-2}$ as a 2-h i.v. infusion; 5-FU $400 \mathrm{mg} \mathrm{m}^{-2}$ i.v. bolus at J1, then $2400 \mathrm{mg} \mathrm{m}^{-2}$ as i.v. infusion over $46 \mathrm{~h}$; and irinotecan $180 \mathrm{mg} \mathrm{m}^{-2}$ as a $1.5-\mathrm{h}$ infusion. Courses were repeated every 2 weeks. Tumour response was assessed according to Response Evaluation Criteria in Solid Tumors (RECIST) criteria and in reviewing computed tomography scans. All adverse events, especially gastrointestinal events and leukopoenia were recorded and graded for severity according to WHO scales. All the patients enrolled had given informed consent to the study, which had been approved by the Comite de protection des personnes dans la recherche biomédicale of Bordeaux.

\section{Pharmacokinetic studies}

For pharmacokinetic studies, blood samples were obtained from the first 28 patients, at the first course of treatment, at 1 and $1.5 \mathrm{~h}$ after the beginning of irinotecan infusion and then at 10, 30, $45 \mathrm{~min}, 1,2,4,8,12,24$ and $48 \mathrm{~h}$ after the end of irinotecan infusion. For the other patients, a limited sampling strategy was adopted and only four samples were obtained, namely at the end, and $10 \mathrm{~min}, 4$ and $24 \mathrm{~h}$ after the end of irinotecan infusion. For the identification of gene polymorphisms, a blood sample was obtained at patient's inclusion, and leukocytes were prepared by Ficoll gradient centrifugation. For the evaluation of the CYP3A4 status, patients received, at least $24 \mathrm{~h}$ before chemotherapy, a dose of $300 \mathrm{mg}$ of cortisol as an i.v. bolus. Urines were collected over $24 \mathrm{~h}$ by fractions of 3,3 and $18 \mathrm{~h}$, and blood samples were obtained 15 and 90 min after cortisol administration. 
Irinotecan and metabolites were evaluated in plasma using an HPLC technique with fluorometric detection, which derives from a technique we developed earlier (Rivory and Robert, 1994). This technique allowed the separation and quantification of irinotecan and all its known metabolites, namely SN-38, SN-38G, APC and NPC. Briefly, $500 \mu \mathrm{l}$ of methanol/1 N hydrochloric acid $(98: 2, \mathrm{v} / \mathrm{v})$ were added to $250 \mu \mathrm{l}$ of plasma. The tubes were vortex-mixed for $10 \mathrm{~s}$, centrifuged at $10000 \mathrm{~g}$ for $5 \mathrm{~min}$ at $4^{\circ} \mathrm{C}$ and $5 \mu \mathrm{l}$ of $1 \mathrm{~N}$ hydrochloric acid was added to $600 \mu \mathrm{l}$ of the supernatant. Later, after centrifugation at $10000 \mathrm{~g}$ for $5 \mathrm{~min}$ at $4^{\circ} \mathrm{C}, 80 \mu \mathrm{l}$ of the supernatant was injected into the HPLC system. For SN-38G estimation, the samples were incubated with $1000 \mathrm{IU}$ of $\beta$-glucuronidase for $2 \mathrm{~h}$ at $37^{\circ} \mathrm{C}$ prior to deproteinisation. The HPLC equipment consists of a Perkin Elmer 200 model with a fluorescence detector (FP-1520, JASCO, Bouguenais, France). Separation of compounds was achieved using a Symmetry Shield RP8 $(5 \mu \mathrm{m}, 150 \times 4.6 \mathrm{~mm}$; Waters, Saint-Quentin-en-Yvelines, France) analytical column protected by a Symmetry Shield RP8 precolumn $(5 \mu \mathrm{m}, 3.9 \times 20 \mathrm{~mm}$; Waters $)$. The mobile phase A was performed with a mixture of $75 \mathrm{~mm}$ ammonium acetate buffer (adjusted to pH 6 with acetic acid)/acetonitrile (85:15, $\mathrm{v} / \mathrm{v})$. The mobile phase $\mathrm{B}$ was acetonitrile. A linear gradient from 0 to $25 \%$ phase $B$ in $30 \mathrm{~min}$ was used at a rate of $1 \mathrm{ml} \mathrm{min}^{-1}$. The fluorescence detector excitation and emission wavelengths were set at 355 and $515 \mathrm{~nm}$, respectively. The limit of quantification was $1 \mu \mathrm{gl}^{-1}$ for all products.

A limited sampling strategy was adopted for the 20 last patients included in the study, based on the study of Canal et al (1996). Individual pharmacokinetic parameters were calculated according to a nonlinear mixed effects approach using NonMem program (Version VI, level I). Irinotecan concentrations were adequately fitted with a three-compartmental model. Posterior Bayesian pharmacokinetic parameters were determined for each patient, especially clearance, which allowed to calculate irinotecan AUC. SN-38 plasma concentrations were analysed by considering irinotecan data and using two additional compartments (i.e., central and peripheral compartment for SN-38). SN-38G and APC concentrations were analysed separately. For both SN-38G and APC, a two-compartment model with first-order input was used. For the validation of the limited sampling strategy, the AUC values of irinotecan and its metabolites were calculated for the 28 data-rich patients using the trapezoidal rule (non-compartmental approach) and compared with those obtained using the population approach.

\section{Metabolic and pharmacogenetic studies}

Cortisol and $6 \beta$-hydroxycortisol were evaluated in urine using an HPLC technique (Rouits et al, 2003). Extraction was performed using an ethyl acetate/isopropanol mixture (85:15, v/v). HPLC was performed, as described earlier, using UV absorbance at $244 \mathrm{~nm}$ for detection.

Genomic DNA was extracted from leukocytes using the QIAamp ${ }^{\circledR}$ DNA minikit from Qiagen or the DNA Isolation Kit from Roche Molecular Diagnostics (Meylan, France). It was quantified by spectrophotometry. Polymerase chain reactions (PCR) were performed on genomic DNA using appropriate primers. Two polymorphisms were sought in genomic DNA: the TA repeat in the UGT1A 1 gene promoter (UGT1A1*28 genotype, rs8175347) and an SNP in the promoter of the CES2 gene $(830 \mathrm{C}>\mathrm{G}$; rs11075646). UGT1A1*28 genotype was determined using the technique of Pyrosequencing (Rouits et al, 2004). The CES2 genotype was determined by sequencing of the amplified PCR products obtained using the following primers: forward: $5^{\prime}$-CTCCTGGGGTCTCCA

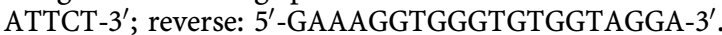

\section{Statistical studies}

Statistics were performed using SPSS software (Chicago, IL, USA). The $\alpha$-error risk was classically chosen as $5 \%$. All statistical tests were bi-directional. Continuous variables were compared using the Pearson coefficient of correlation. Fisher's exact test was used to study the relationships between drug response and toxicity and the parameters studied. Owing to the high number of tests performed, the Bonferroni correction was applied to decrease the probability of detecting falsely positive relationships.

\section{RESULTS}

\section{Clinical outcome}

A total of 49 colorectal cancer patients entered the study; there were 34 males and 15 females and their median age was 60 years, similar for both genders (Table 1). All of them were treated according to the protocol described above, with a total of 190 courses of treatment. Toxicity and overall survival were evaluable for all patients, response for 47 patients and progression-free survival for 35 patients (Table 1). Overall survival was $20 \%$ at 2 years. The median follow up of the patients was 540 days from the onset of chemotherapy.

\section{Pharmacokinetics of irinotecan}

Pharmacokinetic studies were performed during the first course of treatment in 28 patients with 10-13 blood samplings and in 20 additional patients with a limited sampling strategy. The UGT1A1 polymorphism was determined in 44 patients, the CES2 polymorphism in 48 patients and the $6 \beta$-hydroxycortisol/cortisol urinary ratio (measured 3,6 and $24 \mathrm{~h}$ after the administration of $300 \mathrm{mg}$ cortisol) in 46 patients.

The mean pharmacokinetic parameters of irinotecan and its metabolites as obtained using the Bayesian approach are presented in Table 2. Comparison of AUC values obtained using the trapezoidal rule and the population approach was done for the 28 patients with data-rich samplings and revealed coefficients of correlation of $0.96,0.95,0.93$ and 0.99 for irinotecan, SN-38,

Table I Clinical features of the patients entering the study

\begin{tabular}{lc}
\hline Patients included & 49 \\
Gender (M/F) & $34 / 15$ \\
Median age (range) & $60(33-78)$ \\
Performance status $(0 />0)$ & $27 / 20$ \\
Tumour site (colon/rectum/both) & $29 / 16 / 4$ \\
Metastases (hepatic/extrahepatic) & $35 / 14$ \\
Prior adjuvant therapy (yes/no) & $19 / 30$ \\
Prior palliative therapy (yes/no) & $9 / 40$ \\
Overall response (progression/stable disease/objective response) & $10 / 25 / 11$ \\
Neutropoenia over I90 courses (grade I/grade 2/grade $\geqslant 3)$ & $20 / 5 / 9$ \\
Diarrhoea over I90 courses (grade I/grade 2/grade 3) & $38 / 10 / 3$ \\
Median progression-free survival (days) & 92 \\
Median overall survival (months) & 13 \\
\hline
\end{tabular}

Table 2 Pharmacokinetic parameters of irinotecan and metabolites in 48 patients (Bayesian population approach)

\begin{tabular}{lc}
\hline Pharmacokinetic parameters of irinotecan & \\
Total plasma clearance & $31.5 \pm 6.8 \mathrm{Ih}^{-1}$ \\
Volume of distribution at steady state $\left(\mathrm{V}_{\mathrm{ss}}\right)$ & $328 \pm 253 \mathrm{I}$ \\
AUCs of irinotecan and metabolites $\left(\mathrm{mg} \mathrm{h} \mathrm{I}^{-1}\right)$ & \\
Irinotecan & $10530 \pm 27 \mathrm{I3}$ \\
SN-38 & $1133 \pm 337$ \\
SN-38G & $4369 \pm 2165$ \\
APC & $2205 \pm 1293$ \\
\hline
\end{tabular}

Results are given as mean \pm s.d. 
SN-38G and APC, respectively. Bayesian AUC values were used for further correlation analyses.

There was a weakly significant correlation between the dose administered and the AUC values of irinotecan and $\mathrm{SN}-38$ ( $r=0.311$ and 0.265 , respectively, $P=0.03$ and 0.07 ). There were also significant correlations between the AUC of irinotecan and those of each of the metabolites $(0.309<r<0.522,0.0002$ $<P<0.03)$. Only a slight, nonsignificant correlation was found between the AUC values of SN-38 and SN-38G $(r=0.211, P<0.15)$. A highly significant correlation existed between the AUC values of APC and NPC $\left(r=0.857, P<10^{-7}\right)$. It is also remarkable that the relative extent of glucuronidation of $\mathrm{SN}-38$ (as defined by the ratio of the AUC of SN-38G to that of SN-38, see Rivory et al, 1997) was inversely correlated with the relative extent of the activation of irinotecan (as defined by the ratio of the AUC of SN-38 to that of irinotecan) $(r=-0.426, P=0.002)$.

\section{Relationships between clinicobiological and pharmacokinetic parameters}

No relationship between treatment response, progression-free survival or toxicity and any of the pharmacokinetic parameters was evidenced. In contrast, several important biological constants were significantly correlated to pharmacokinetic parameters. Limited to the significant relationships after Bonferroni correction, we observed that the pretreatment leukocyte and granulocyte counts were significantly correlated with SN-38 AUC (or the relative extent of activation) $(r=0.402, P=0.004$, Figure $2 \mathrm{~A})$, that a series of hepatic parameters (alkaline phosphatase, transaminases and lactate dehydrogenase) were significantly correlated with the AUC values of APC (or the relative extent of metabolisation) $\left(0.456<r<0.576,2 \times 10^{-5}<P<0.0001\right.$, Figure $\left.2 \mathrm{~B}\right)$ and that plasma creatinine was significantly correlated (or creatinine clearance inversely correlated) with the AUC value of SN-38G (or the relative extent of glucuronidation) $(0.318<r<0.412$, $0.004<P<0.03$, Figure 2C)

\section{Relationships between pharmacokinetic parameters and metabolic predictors}

The rare allele (seven TA repeats) frequency of UGT1A1 was 0.30 and the rare allele frequency $(\mathrm{G})$ of $C E S 2$ was 0.14 . Both genotype distributions followed the Hardy-Weinberg equilibrium. There was no relationship between the polymorphisms of UGT1A1 or CES2 and any of the pharmacokinetic parameters obtained, the mean values of each parameter being not significantly different in wild-type or variant patients for either polymorphism. The relationship between UGT1A1 polymorphism and SN-38G AUC value especially failed to reach significance $(P=0.20)$. In contrast, there was a consistent association between the $6 \beta$-hydroxycortisol/ cortisol ratio at $6 \mathrm{~h}$ after cortisol injection and the AUC values of APC (or the relative extent of metabolisation) $(r=0.437$, $P=0.002)$.

\section{Relationships between clinicobiological parameters and metabolic predictors}

The relationships between the three metabolic predictors (UGT1A1 and CES2 polymorphisms and the $6 \beta$-hydroxycortisol/cortisol ratio) and the clinical and biological parameters could be studied in the whole set of patients, independent of the pharmacokinetic data. The UGT1A1 polymorphism was significantly related to the bilirubin plasma level: variant homozygous patients had a mean bilirubin plasma level of 2.5 higher than heterozygous $\left(P=3 \times 10^{-6}\right)$ or common homozygous patients $\left(P=7 \times 10^{-7}\right)$ (Table 3). This was also the case for ALT transaminase when comparing common and variant homozygous patients $(P=0.004)$. Variant homozygous patients also had significantly lower plasma
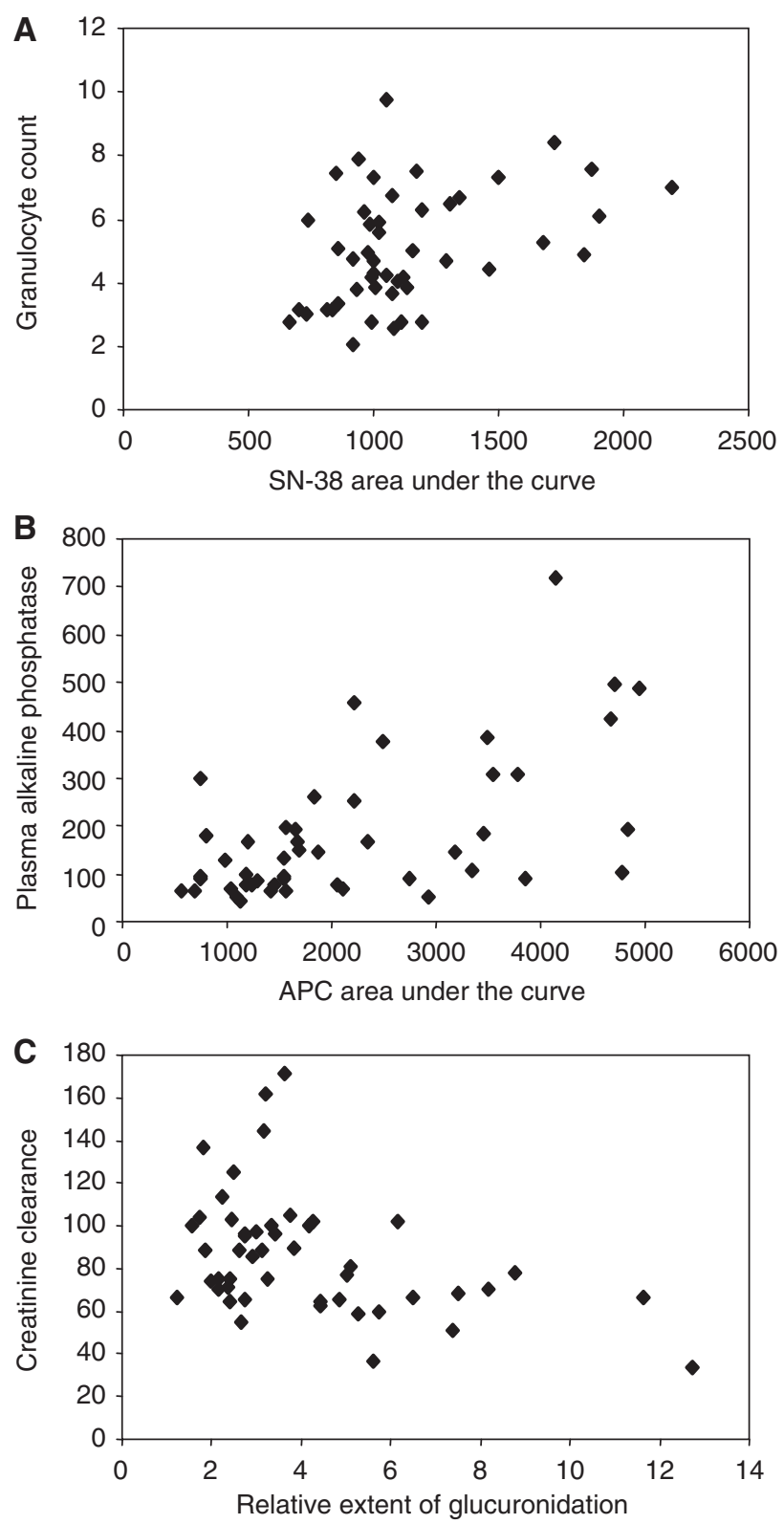

(SN-38G/SN-38)

Figure 2 Charts representing the correlations between $(\mathbf{A}) \mathrm{SN}-38 \mathrm{AUC}$ value $\left(\mathrm{mg} \mathrm{h}^{-1} \mathrm{I}^{-1}\right)$ and pretreatment granulocyte counts $\left(\times 10^{9}\right) r=0.402$, $P=0.005$; (B) APC AUC value $\left(\mathrm{mg} \mathrm{h}^{-1} \mathrm{I}^{-1}\right)$ and pretreatment plasma alkaline phosphatase $\left(\mathrm{IUI}^{-1}\right) r=0.576, P=2 \times 10^{-5}$; (C) Relative extent of glucuronidation (SN-38G/SN-38) and pretreatment creatinine clearance $\left(\mathrm{ml} \mathrm{min}{ }^{-1}\right) r=0.412, P=0.004$

sodium levels than heterozygous or common homozygous patients, but the difference was only $3.6 \%(P=0.001)$. This polymorphism was a significant predictor of toxicity: 3 patients out of 23 common homozygous underwent an episode of grade $>1$ neutropoenia during at least one of the four first courses of treatment, whereas this was the case for 9 patients out of 21 heterozygous or variant homozygous $(P=0.042$ with Fisher's exact test). No relationship could be evidenced between UGT1A1 polymorphism and treatment response- or progression-free survival (Table 3 ).

The CES2 polymorphism was unrelated to any of the biological parameters analysed, with the exception of plasma lactate dehydrogenase, which was twice lower in common homozygous 
Table 3 Relationships between the metabolic predictors and efficacy and toxicity of the treatment

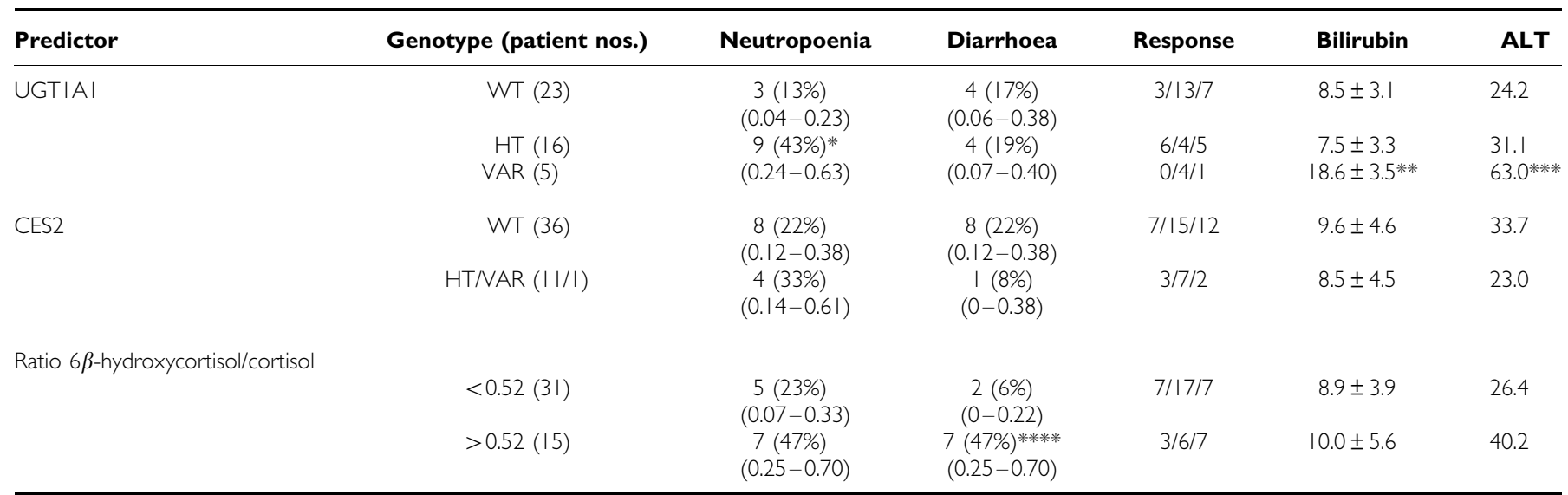

For UGTIAI and CES2, the genotypes were homozygous wild type (WT), heterozygous (HT) or homozygous variant (VAR). For the CYP3A4 status, the ratio $6 \beta$-hydoxycortisol/cortisol was split at a threshold of 0.52 , as described in the article. The number of patients in each group is indicated in text. For toxicity data, the number of patients undergoing grade $>2$ toxicity (WHO grading) is indicated, with the corresponding percentage between parentheses and the $95 \%$ confidence intervals between parentheses. For response, the number of patients with progression/stabilisation/objective response is indicated, using RECIST criteria. Mean bilirubin ( $\mu$ mol $I^{-}$) and $A L T$ values $\left(\mathrm{UI} \mathrm{I}^{-1}\right)$ for each group of patients are indicated. Asterisks indicate a significant difference between patients' groups: $* P=0.042 ; * * P=3 \times 10^{-6} ; * * * P=0.004 ;$ and $* * * * P=0.03$.

patients than in patients having at least one variant allele $(P=0.02$, not significant after the Bonferroni correction). This polymorphism was unrelated to treatment response or progression-free survival, but appeared as a modest predictor of diarrhoea: 8 patients out of 36 common homozygous underwent an episode of grade $>1$ diarrhoea during at least one of the four first courses of treatment, whereas this was the case for 1 patient out of 12 patients having at least one variant allele (not significant) (Table 3 ).

The urinary $6 \beta$-hydroxycortisol/cortisol ratio was studied at three different time intervals after the administration of $300 \mathrm{mg}$ cortisol. There was a significant positive correlation between the 6 -h ratio and the plasma transaminases level $(r=0.501$, $P=0.0003)$. There was a significant association between the $6-\mathrm{h}$ (and the 24-h) ratio and the risk of grade $>1$ toxicity: patients who had encountered such an episode of neutropoenia or diarrhoea during any of the four first courses of treatment had a 1.84-fold higher 6-h ratio than patients who had not $(P=0.022)$. From these data, it was possible to define a threshold above which the risk of toxicity is maximal; using the method of Youden, it was possible to optimise this threshold at 0.52 , giving a sensitivity of $80 \%$ and a specificity of $77 \%$. The association between a high 6 -h ratio and the risk of toxicity is especially marked for diarrhoea: only 2 patients out of 31 with a ratio $<0.52$ underwent grade $>1$ diarrhoea, whereas this was the case for 8 patients out of 15 with a ratio $>0.52(P=0.003$, with the Fisher exact test) (Table 3$)$.

\section{DISCUSSION}

The mean pharmacokinetic and metabolic parameters we obtained for the 49 patients by Bayesian analysis were within the range of the values usually obtained (Mathijssen et al, 2003) and the dispersion of the values is also comparable. The correlation between the dose of irinotecan administered and the AUCs of both irinotecan and SN-38 has been observed in most pharmacokinetic studies. The correlation between APC and NPC AUCs was expected in view of the fact that these two metabolites are produced by the same enzyme, CYP3A4, in two parallel pathways (Haaz et al, $1998 \mathrm{a}, \mathrm{b})$. However, the inverse correlation between the relative extent of SN-38 glucuronidation and the relative extent of irinotecan activation has never been mentioned in the published pharmacokinetic studies. In other words, a high glucuronidation rate is associated with a low rate of formation of SN-38. This would tend to indicate that the formation of the glucuronide is independent of the availability of SN-38 and that a fixed amount of $\mathrm{SN}-38$ is transformed into $\mathrm{SN}-38 \mathrm{G}$, suggesting a saturation process.

There was no direct relationship between the pharmacokinetic parameters and the clinical outcome, both for toxicity and efficacy. Such pharmacokinetic - pharmacodynamic relationships have been found in some studies (de Forni et al, 1994; Abigerges et al, 1995; Sasaki et al, 1995; Canal et al, 1996) but not all (Catimel et al, 1995; Rothenberg et al, 1996; Herben et al, 1999; Ma et al, 2000). For instance, neutropoenia and diarrhoea could be predicted from irinotecan and SN-38 AUCs in three original studies (Abigerges et al, 1995; Sasaki et al, 1995; Canal et al, 1996). However, such correlations were obtained in early dose-finding studies with a large range of doses prescribed and, therefore, a large range of AUC values. In phase III studies performed at a fixed dose, the AUC range is usually too small to identify pharmacokineticpharmacodynamic relationships.

A careful analysis of the relationships between the pharmacokinetic parameters and the pretreatment biological constants has revealed several important features. The leukocyte count was associated with SN-38 AUC, which would suggest that a significant part of the plasma SN-38 might originate from blood-nucleated cells. The role of leukocytes in the activation of irinotecan has been mentioned in a study of Cecchin et al (2005) who observed an association between CES2 mRNA expression in peripheral blood mononuclear cells and the level of irinotecan activation in patients' plasma. The correlation between the level of conversion of cortisol into $6 \beta$-hydroxycortisol, and the plasma levels of APC is in agreement with the major role of CYP3A4 in this pathway of transformation of irinotecan (Haaz et al, 1998b). The correlation between plasma liver enzymes and the AUC of APC was unexpected, as it has been shown that CYP3A activity was reduced by $50 \%$ in patients with concurrent elevations in liver transaminases (Baker et al, 2004); however, it has also been shown that CYP3A4 expression was associated with the occurrence of metastases in osteosarcoma (Dhaini et al, 2003), which might explain why patients with high cortisol conversion into $6 \beta$-hydroxycortisol had altered liver enzymes. Another interesting observation is that creatinine clearance was inversely correlated with SN-38G AUC. This would indicate that renal function plays a major role in the disposition of SN-38G and that the elimination of this metabolite is a determinant of its plasma concentration as important as its formation from SN-38. 
The UGT1A1*28 polymorphism did not appear to be significantly related to the SN38-G AUC value or to the extent of glucuronidation as has been observed in other studies (Iyer et al, 2002; Rouits et al, 2004; Toffoli et al, 2006; Côté et al, 2007; Ramchandani et al, 2007) whereas, as expected, the bilirubin plasma levels were much higher in the variant homozygous patients than in common homozygous and heterozygous patients. Despite the absence of significant association with the glucuronidation rate of $\mathrm{SN}-38$, the UGT1A1 polymorphism appeared to be significantly associated with irinotecan toxicity, the patients having at least one variant allele being at increased risk of neutropoenia. This has been already shown to be in relation with the decreased transcription rate of UGT1A1 when the promoter harbours seven TA repeats, and to the subsequent decrease of enzyme dosage for SN-38 detoxification. Our study thus confirms the importance of the pre-therapeutic determination of the UGT1A1 genotype for predicting irinotecan toxicity, and indicates that heterozygous patients may be also at increased risk for neutropoenia. Bilirubinemia by itself did not appear as a predicting factor for irinotecan toxicity.

It is remarkable that the CYP3A4 status, defined by the $6 \beta$-hydoxycortisol/cortisol ratio, appears as a predictor of the diarrhoea and, to a lesser extent, to the neutropoenia undergone by the patients, which is an original and important observation. Few studies, until recently, have evaluated the pharmacokinetics of these metabolites, and no study establishing metabolicpharmacodynamic relationship is presently available. The mechanism by which the CYP3A4 status influences the occurrence of irinotecan-induced diarrhoea remains elusive, as the metabolites of irinotecan produced in this pathway do not display cytotoxic activity. It might be simply related to the hepatic dysfunction that is associated with high cortisol biotransformation in this group of patients. Nevertheless, it appears tempting to propose the use of a pretreatment CY3A4 determination for predicting this major side effect of irinotecan, using, for instance, the cortisol $6 \beta$-hydroxylation approach or another one, such as the determination of midazolam clearance, which has been shown to be significantly associated with irinotecan clearance (Mathijssen et al, 2004).

As a conclusion, our studies have revealed several interesting tracks concerning the prediction of irinotecan toxicity and some new insights on the metabolism - pharmacodynamic relationships of this major drug. We confirm the use of UGT1A1 genotyping to predict for haematological toxicity, both for homozygous and heterozygous patients; we demonstrate also the potential importance of CYP3A4 status to predict for the occurrence of digestive toxicity. We show in addition that SN-38G plasma levels are associated with renal function, that the participation of blood cells in irinotecan activation might be more important than previously thought and that the extent of glucuronidation of $\mathrm{SN}-38$ is inversely related to the relative formation of $\mathrm{SN}-38$ from irinotecan. All these observations may improve the routine use of irinotecan in colorectal cancer patients.

\section{ACKNOWLEDGEMENTS}

This work was supported by grants from Aventis and Pfizer. The continuous support of the French League against Cancer (comite de Charente-Maritime) is gratefully acknowledged.

\section{REFERENCES}

Abigerges D, Chabot GG, Armand JP, Herait P, Gouyette A, Gandia D (1995) Phase I and pharmacologic studies of the camptothecin analog irinotecan administered every 3 weeks in cancer patients. J Clin Oncol 13: $210-221$

André T, Louvet C, Maindrault-Goebel F, Couteau C, Mabro M, Lotz JP, Gilles-Amar V, Krulik M, Carola E, Izrael V, de Gramont A (1999) CPT-11 (irinotecan) addition to bimonthly, high-dose leucovorin and bolus and continuous-infusion 5-fluorouracil (FOLFIRI) for pretreated metastatic colorectal cancer. Eur J Cancer 35: 1343-1347

Baker SD, van Schaik RH, Rivory LP, Ten Tije AJ, Dinh K, Graveland WJ, Schenk PW, Charles KA, Clarke SJ, Carducci MA, McGuire WP, Dawkins F, Gelderblom H, Verweij J, Sparreboom A (2004) Factors affecting cytochrome P-450 3A activity in cancer patients. Clin Cancer Res 10: $8341-8350$

Bentrem DJ, Dematteo RP, Blumgart LH (2005) Surgical therapy for metastatic disease to the liver. Annu Rev Med 56: 139-156

Boyle P, Ferlay J (2005) Cancer incidence and mortality in Europe, 2004. Ann Oncol 16: 481-488

Canal P, Gay C, Dezeuze A, Douillard JY, Bugat R, Brunet R, Adenis A, Hérait P, Lokiec F, Mathieu-Boué A (1996) Pharmacokinetics and pharmacodynamics of irinotecan during a phase II clinical trial in colorectal cancer. J Clin Oncol 14: 2688-2695

Catimel G, Chabot GG, Guastalla JP, Dumortier A, Cote C, Engel C, Gouyette A, Mathieu-Boue A, Mahjoubi M, Clavel M (1995) Phase I and pharmacokinetic study of irinotecan (CPT-11) administered daily for three consecutive days every three weeks in patients with advanced solid tumors. Ann Oncol 6: 133-140

Cecchin E, Corona G, Masier S, Biason P, Cattarossi G, Frustaci S, Buonadonna A, Colussi A, Toffoli G (2005) Carboxylesterase isoform 2 mRNA expression in peripheral blood mononuclear cells is a predictive marker of the irinotecan to SN38 activation step in colorectal cancer patients. Clin Cancer Res 11: 6901-6907

Charasson V, Bellott R, Meynard D, Longy M, Gorry P, Robert J (2004) Pharmacogenetics of human carboxylesterase 2, an enzyme involved in the activation of irinotecan into SN-38. Clin Pharmacol Ther 76: 528-535

Côté JF, Kirzin S, Kramar A, Mosnier JF, Diebold MD, Soubeyran I, Thirouard AS, Selves J, Laurent-Puig P, Ychou M (2007) UGT1A1 polymorphism can predict hematologic toxicity in patients treated with irinotecan. Clin Cancer Res 13: 3269-3275

Cunningham D, Humblet Y, Siena S, Khayat D, Bleiberg H, Santoro A, Bets D, Mueser M, Harstrick A, Verslype C, Chau I, Van Cutsem E (2004) Cetuximab monotherapy and cetuximab plus irinotecan in irinotecanrefractory metastatic colorectal cancer. $N$ Engl J Med 351: 337 - 345

de Forni M, Bugat R, Chabot GG, Culine S, Extra JM, Gouyette A, Madelaine I, Marty ME, Mathieu-Boué A (1994) Phase I and pharmacokinetic study of the camptothecin derivative irinotecan, administered on a weekly schedule in cancer patients. Cancer Res 54: 4347-4354

de Gramont A, Figer A, Seymour M, Homerin M, Hmissi A, Cassidy J, Boni C, Cortes-Funes H, Cervantes A, Freyer G, Papamichael D, Le Bail N, Louvet C, Hendler D, de Braud F, Wilson C, Morvan F, Bonetti A (2000) Leucovorin and fluorouracil with or without oxaliplatin as first-line treatment in advanced colorectal cancer. J Clin Oncol 18: 2938-2947

Dhaini HR, Thomas DG, Giordano TJ, Johnson TD, Biermann JS, Leu K, Hollenberg PF, Baker LH (2003) Cytochrome P450 CYP3A4/5 expression as a biomarker of outcome in osteosarcoma. J Clin Oncol 21: 2481-2485 Douillard JY, Cunningham D, Roth AD, Navarro M, James RD, Karasek P, Jandik P, Iveson T, Carmichael J, Alakl M, Gruia G, Awad L, Rougier P (2000) Irinotecan combined with fluorouracil compared with fluorouracil alone as first-line treatment for metastatic colorectal cancer: a multicentre randomised trial. Lancet 355: 1041-1047

Haaz MC, Riché C, Rivory LP, Robert J (1998a) Biosynthesis of an aminopiperidino metabolite of irinotecan (7-ethyl-10-(4-(1-piperidino)1-piperidino)carbonyloxycamptothecine) by human hepatic microsomes. Drug Metab Dispos 26: 769-774

Haaz MC, Rivory L, Riché C, Vernillet L, Robert J (1998b) Metabolism of irinotecan (CPT-11) by human hepatic microsomes: participation of cytochrome P-450 3A and drug interactions. Cancer Res 58: 468-472

Herben VM, Schellens JH, Swart M, Gruia G, Vernillet L, Beijnen JH, ten Bokkel Huinink WW (1999) Phase I and pharmacokinetic study of irinotecan administered as a low-dose, continuous intravenous infusion 
over 14 days in patients with malignant solid tumors. J Clin Oncol 17: $1897-1905$

Hill C, Doyon F (2005) The frequency of cancer in France in year 2000, and trends since 1950. Bull Cancer 92: 7-11

Iyer L, Das S, Janisch L, Wen M, Ramirez J, Karrison T, Fleming GF, Vokes EE, Schilsky RL, Ratain MJ (2002) UGT1A1*28 polymorphism as a determinant of irinotecan disposition and toxicity. Pharmacogenomics $J$ 2: $43-47$

Labianca R, Pessi MA, Zamparelli G (1997) Treatment of colorectal cancer. Current guidelines and future prospects for drug therapy. Drugs 53: $593-607$

Lamba JK, Lin YS, Schuetz EG, Thummel KE (2002) Genetic contribution to variable human CYP3A-mediated metabolism. Adv Drug Deliv Rev 54: $1271-1294$

Ma MK, Zamboni WC, Radomski KM, Furman WL, Santana VM, Houghton PJ, Hanna SK, Smith AK, Stewart CF (2000) Pharmacokinetics of irinotecan and its metabolites SN-38 and APC in children with recurrent solid tumors after protracted low-dose irinotecan. Clin Cancer Res 6: 813-819

Mathijssen RH, de Jong FA, van Schaik RH, Lepper ER, Friberg LE, Rietveld T, de Bruijn P, Graveland WJ, Figg WD, Verweij J, Sparreboom A (2004) Prediction of irinotecan pharmacokinetics by use of cytochrome P450 3A4 phenotyping probes. J Natl Cancer Inst 96: 1585-1592

Mathijssen RH, Marsh S, Karlsson MO, Xie R, Baker SD, Verweij J, Sparreboom A, McLeod HL (2003) Irinotecan pathway genotype analysis to predict pharmacokinetics. Clin Cancer Res 9: 3246-3253

Moses MA, Harper J, Fernandez CA (2004) A role for antiangiogenic therapy in breast cancer. Curr Oncol Rep 6: $42-48$

Ramchandani RP, Wang Y, Booth BP, Ibrahim A, Johnson JR, Rahman A, Mehta M, Innocenti F, Ratain MJ, Gobburu JV (2007) The role of SN-38 exposure, UGT1A1*28 polymorphism, and baseline bilirubin level in predicting severe irinotecan toxicity. J Clin Pharmacol 47: 78-86

Rivory LP, Haaz MC, Canal P, Lokiec F, Armand JP, Robert J (1997) Pharmacokinetic interrelationships of irinotecan (CPT-11) and its three major plasma metabolites in patients enrolled in phase I/II trials. Clin Cancer Res 3: 1261 - 1266

Rivory LP, Robert J (1994) A reverse-phase HPLC method for the simultaneous quantification of the carboxylate and lactone forms of the camptothecin derivative irinotecan (CPT-11) and of its metabolite SN-38 in plasma. J Chromatogr B 661: $133-141$

Rivory LP, Robert J (1995) Molecular, cellular, and clinical aspects of the pharmacology of 20(S)camptothecin and its derivatives. Pharmacol Ther 68: $269-296$

Rothenberg ML, Eckardt JR, Kuhn JG, Burris III HA, Nelson J, Hilsenbeck SG, Rodriguez GI, Thurman AM, Smith LS, Eckhardt SG, Weiss GR, Elfring GL, Rinaldi DA, Schaaf LJ, Von Hoff DD (1996) Phase II trial of irinotecan in patients with progressive or rapidly recurrent colorectal cancer. J Clin Oncol 14: 1128-1135

Rouits E, Boisdron-Celle M, Dumont A, Guerin O, Morel A, Gamelin E (2004) Relevance of different UGT1A1 polymorphisms in irinotecaninduced toxicity: a molecular and clinical study of 75 patients. Clin Cancer Res 10: $5151-5159$

Rouits E, Boisdron-Celle M, Morel A, Gamelin E (2003) Simple and sensitive high-performance liquid chromatography method for simultaneous determination of urinary free cortisol and $6 \beta$-hydroxycortisol in routine practice for CYP 3A4 activity evaluation in basal conditions and after grapefruit juice intake. J Chromatogr B 793: 357 - 366

Sasaki Y, Hakusui H, Mizuno S, Morita M, Miya T, Eguchi K, Shinkai T, Tamura T, Ohe Y, Saijo N (1995) A pharmacokinetic and pharmacodynamic analysis of CPT-11 and its active metabolite SN-38. Jpn J Cancer Res 86: $101-110$

Taieb J, Artru P, Paye F, Louvet C, Perez N, Andre T, Gayet B, Hebbar M, Goebel FM, Tournigand C, Parc R, de Gramont A (2005) Intensive systemic chemotherapy combined with surgery for metastatic colorectal cancer: results of a phase II study. J Clin Oncol 23: 502-509

Toffoli G, Cecchin E, Corona G, Russo A, Buonadonna A, D'Andrea M, Pasetto LM, Pessa S, Errante D, De Pangher V, Giusto M, Medici M, Gaion F, Sandri P, Galligioni E, Bonura S, Boccalon M, Biason P, Frustaci $S(2006)$ The role of UGT1A1*28 polymorphism in the pharmacodynamics and pharmacokinetics of irinotecan in patients with metastatic colorectal cancer. J Clin Oncol 24: $3061-3068$

Yamamoto N, Tamura T, Kamiya Y, Sekine I, Kunitoh H, Saijo N (2000) Correlation between docetaxel clearance and estimated cytochrome P450 activity by urinary metabolite of exogenous cortisol. J Clin Oncol 18: $2301-2308$ 